\title{
Carnets
}

Revue électronique d'études françaises de l'APEF

Deuxième série - 13 | 2018

Corps, rythmes et voix : en/jeux littéraires et artistiques

\section{La voix contemporaine de Molière (ou les voies d'un Impromptu sur scène)}

\section{Marta Teixeira Anacleto}

\section{CpenEdition}

Journals

Édition électronique

URL : http://journals.openedition.org/carnets/2609

DOI : $10.4000 /$ carnets.2609

ISSN : 1646-7698

Éditeur

APEF

Référence électronique

Marta Teixeira Anacleto, «La voix contemporaine de Molière (ou les voies d'un Impromptu sur scène) », Carnets [En ligne], Deuxième série - 13 | 2018, mis en ligne le 31 mai 2018, consulté le 19 avril 2019.

URL : http://journals.openedition.org/carnets/2609 ; DOI : 10.4000/carnets.2609

Ce document a été généré automatiquement le 19 avril 2019

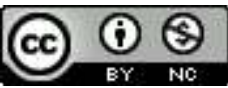

Carnets est mis à disposition selon les termes de la licence Creative Commons - Atribution - Pas d'utilisation commerciale 4.0 International. 


\title{
La voix contemporaine de Molière (ou les voies d'un Impromptu sur scène)
}

\author{
Marta Teixeira Anacleto
}

1 «Le théâtre est le désir qu'a la parole de se faire corps (...). Ce n'est pas le corps qui s'approprie la parole, mais la parole qui s'approprie un corps - un corps éphémère, celui de l'acteur, la parole demeurant immuable, quand bien même l'acteur essaierait de l'adapter et de la façonner à son corps » (Júdice, $2006: 11^{1}$ ). La phrase citée fait partie de la définition du théâtre qui ouvre la préface écrite par Nuno Júdice, «La parole et le mythe ", pour sa traduction de Dom Juan de Molière (Molière, 2006), destinée à la mise-enscène portugaise de Ricardo Pais, représentée en 2006 au Théâtre National S. João de Porto. Le même propos théorique aurait pu être énoncé, en 2016, par le traducteur de L'Impromptu de Versailles (Molière, 2016), João Paulo Esteves da Silva, dont le travail de réécriture a permis à Miguel Loureiro de mettre en scène cette pièce courte et polémique de Molière au Théâtre National D. Maria II, à Lisbonne. En fait, la voix contemporaine de Molière renvoie, presque tout le temps, dans ces deux temps en tout cas, à un déplacement historique de la parole théatrale créé par le dramaturge, à Versailles, vers des voies esthétiques relevant d'une méta-théâtralité implicite aux textes du XVII et à ceux du $\mathrm{xxI}^{\mathrm{e}}$, en conciliant la voix de l'auteur, celle des metteurs-en-scène et celle des acteurs.

2 C'est ainsi que, dans le contexte de cette polyphonie, le discours théâtral de Molière s'avère, de nos jours, redevable d'une définition utopique de la scène, celle-ci étant le lieu, comme l'ont très bien compris le dramaturge de Louis XIV, Ricardo Pais et Miguel Loureiro, où le corps et la parole assimilent l'écriture. Ne serait-ce que par la force des textes en contexte (L'Impromptu s'encadre dans «La Querelle de l'École des Femmes » 1663 -, Dom Juan dans la célèbre "Querelle du Tartuffe » - 16652), l'écriture dramatique s'impose, dans les deux cas, par l'intégration d'une poétique de la voix forgée par l'auteur pour répondre à ses détracteurs - c'est-à-dire comme une façon de dire le théâtre et d'en faire un instrument politique et éthique. En fait, si Molière s'est servi de la scène pour 
attaquer, en gestes et en paroles, le théâtre affecté des acteurs de l'hôtel de Bourgogne, ou la cabale des faux dévots, les deux metteurs-en-scène portugais se proposent, eux, de transposer cette théâtralité auto-réfléchie (un impromptu créé à Versailles ; un Dom Juan histrionique faisant l'hypocrite) sur la scène contemporaine, là où le théâtre exhibe ses artifices et s'exhibe par la matérialité de l'écriture.

Il semble donc légitime de définir deux axes d'expressivité actuelle de la voix de Molière, lui-même auteur et acteur d'un continuel « impromptu » métaphorique : la voix du texte et la voix de la scène. La synthèse des deux se situe, visiblement, dans un déplacement historique des discours ${ }^{3}$ qui prolonge l'ouverture axiale des textes originaux - une répétition avortée dans une salle de Versailles, la mort spectaculaire d'un libertin.

\section{La voix du texte (ou Molière par lui-même)}

Selon Nuno Júdice, traducteur portugais de Dom Juan, au théâtre, la voix s'approprie un corps, le corps éphémère de l'acteur (Júdice, 2006: 11). Dans L'Impromptu de Versailles, texte représenté en 1663 pour le Roi, au moment où Molière devient son dramaturge officiel, la voix de l'auteur correspond à la voix du personnage-acteur Molière, «marquis ridicule » (Molière, $1965: 145$ ), suivant un dédoublement biographique qui s'intègre dans la "Querelle de l'École des Femmes": les personnages dramatiques se transforment en «acteurs» pour répondre aux critiques des acteurs de l'Hôtel de Bourgogne qui dénonçaient l'invraisemblance éthique de la pièce, pour critiquer leur style artificiel, et pour régler son compte à Boursault, auteur d'une comédie jouée dans cette salle de spectacles parisienne - Le Portrait du peintre -, qui parodie La Critique de l'École des Femmes ${ }^{4}$ :

MADEMOISELLE BÉJART - Mais puisqu'on vous a commandé de travailler sur le sujet de la critique qu'on a faite contre vous, que n'avez-vous fait cette comédie des comédiens dont vous nous avez parlé il y a longtemps? C'était une affaire toute trouvée, et qui venait fort bien à la chose, et d'autant mieux, qu'ayant entrepris de vous peindre, ils vous ouvraient l'occasion de les peindre aussi, et que cela aurait pu s'appeler leur portrait, à bien plus juste titre que tout ce qu'ils ont fait ne peut être appelé le vôtre ; car vouloir contrefaire un comédien dans un rôle comique, ce n'est pas le peindre lui-même, c'est peindre d'après lui les personnages qu'il représente, et se servir des mêmes traits et des mêmes couleurs, qu'il est obligé d'employer aux différents tableaux des caractères ridicules, qu'il imite d'après nature. Mais contrefaire un comédien dans des rôles sérieux, c'est le peindre par des défauts qui sont entièrement de lui, puisque ces sortes de personnages ne veulent, ni les gestes, ni les tons de voix ridicules, dans lesquels on le reconnaît.

MOLIÈRE - Il est vrai, mais j'ai mes raisons pour ne le pas faire, et je n'ai pas cru entre nous que la chose en valût la peine, et puis il fallait plus de temps pour exécuter cette idée. Comme leurs jours de comédies sont les mêmes que les nôtres, à peine ai-je été les voir, que trois ou quatre fois depuis que nous sommes à Paris, je n'ai attrapé de leur manière de réciter, que ce qui m'a d'abord sauté aux yeux, et j'aurais eu besoin de les étudier davantage pour faire des portraits bien ressemblants (Molière, $1965: 150$ ).

Ce jeu expérimental où Molière, dramaturge et acteur de lui-même, fait représenter une répétition incomplète (ou fausse) d'une pièce commandée par le Roi ${ }^{5}$, met en évidence des mécanismes méta-théâtraux d'une étonnante modernité: l'auteur-acteur distribue les différents rôles à ses comédiens, leur apprend à jouer une pièce sous forme de " comédie des comédiens ", les oriente sur la bonne façon de jouer, révélant son adhésion à une écriture spéculaire dont le sujet principal est le théâtre, la voix de l'auteur (Molière dramaturge, Molière-acteur jouant le marquis ridicule) et les voix des acteurs (incarnant 
les rôles paradigmatiques des ridicules moliéresques - le marquis fâcheux, la coquette, la servante précieuse, le poète pédant) :

MOLIÈRE. - Cela est vrai, et c'est en quoi vous faites mieux voir que vous êtes excellente comédienne de bien représenter un personnage, qui est si contraire à votre humeur : tâchez donc de bien prendre tous le caractère de vos rôles, et de vous figurer que vous êtes ce que vous représentez.

(À $d u$ Croisy.) Vous faites le poète, vous, et vous devez vous remplir de ce personnage, marquer cet air pédant qui se conserve parmi le commerce du beau monde, ce ton de voix sentencieux, et cette exactitude de prononciation qui appuie sur toutes les syllabes, et ne laisse échapper aucune lettre de la plus sévère orthographe.

(À Brécourt.) Pour vous, vous faites un honnête homme de cour, comme vous avez déjà fait dans La Critique de l'Ecole des femmes, c'est-à-dire que vous devez prendre un air posé, un ton de voix naturel, et gesticuler le moins qu'il vous sera possible. (À de la Grange.) Pour vous je n'ai rien à vous dire.

(À Mademoiselle Béjart.) Vous, vous représentez une de ces femmes, qui pourvu qu'elles ne fassent point l'amour, croient que tout le reste leur est permis, de ces femmes qui se retranchent toujours fièrement sur leur pruderie, regardent un chacun de haut en bas, et veulent que toutes les plus belles qualités que possèdent les autres, ne soient rien en comparaison d'un misérable honneur dont personne ne se soucie, ayez toujours ce caractère devant les yeux pour en bien faire les grimaces.

(À Mademoiselle de Brie.) Pour vous, vous faites une de ces femmes qui pensent être les plus vertueuses personnes du monde, pourvu qu'elles sauvent les apparences, de ces femmes qui croient que le péché n'est que dans le scandale, qui veulent conduire doucement les affaires qu'elles ont sur le pied d'attachement honnête, et appellent amis ce que les autres nomment galants, entrez bien dans ce caractère. (Molière, 1965 : 153-154).

6 Un impromptu conçu à Versailles désigne, ainsi, de prime abord, une répétition feinte mais néanmoins écrite et représentée d'" après nature ", pendant laquelle les acteurs réagissent aux conseils de l'auteur-acteur sur l'écriture théâtrale et l'art de la représentation (diction et intonation comprises) ${ }^{6}$. L'impromptu incorpore, par ailleurs, des enjeux mimétiques, le réel et l'artificiel se confondant dans la structure du théâtre dans le théâtre que Molière reproduit par la citation continuelle de son École des Femmes et de sa Critique de l'École des Femmes, à laquelle s'ajoute, à la scène $\mathrm{V}^{7}$, la citation du Portrait $d u$ peintre de Boursault, réplique de la Critique. Cette confluence complexe des voix et des corps se décline, à vrai dire, dans la construction d'une "forme-texte » originale et moderne : la répétition à jamais accomplie qui prépare le grand spectacle situé au-delà de la pièce-impromptu; la mise en abyme d'une comédie (L'École), questionnée à l'intérieur d'une autre ( $(a$ Critique), pour faire valoir une poétique théâtrale associée à l'esthétique du ridicule.

C'est justement ce principe vertigineux de l'encadrement, l'« esthétique du miroitement » et la «posture ironique » (Dandrey, 2014: 351-358) à partir desquels Molière interroge l'art de la scène dans une perspective satirique et critique, que Miguel Loureiro reprend pour mettre sur scène son Impromptu (qui ne cesse d'être aussi celui de Molière). Le ton ironique de la voix de l'auteur-acteur, voire la réécriture double constamment exhibée dans une pièce sans sujet (ou dont le sujet est le théâtre dans le théâtre ${ }^{8}$ ), sont le point de départ majeur d'une mise-en-scène contemporaine qui ne cesse de souligner la distance esthétique créée dans une salle imaginée de Versailles. En fait, c'est à partir du contexte et de l'univers du texte de Molière («la voix du texte» en tant que prétexte métathéâtral), que Miguel Loureiro s'affirme, en 2016, comme metteur en scène d'un Théâtre 
National, pour montrer aux spectateurs sa manière de penser le théâtre, dédoublant les impromptus, les effets d'improvisation depuis le début du spectacle : avant d'introduire le texte de Molière, dans un prologue inventé, deux femmes dialoguent hors scène, face à face, dans des loges, citant des extraits du Paradoxe du Comédien de Diderot, pour souligner la nature complexe du pacte théâtral. De même, à partir de la poétique explicite de L'Impromptu et de son inachèvement tacite (la pièce qui n'est jamais représentée), le metteur en scène portugais introduit, dans le texte original traduit, d'autres textes de dramaturges et de théoriciens du théatre, tels que Corneille, Racine, Diderot, Eschyle, qui, à un rythme hallucinant, multiplient les voix sur scène et prolongent l'effet d'autoréflexivité d'une pièce interrompue sans arrêt par les discussions des acteurs. Loureiro crée de constants niveaux d'enchâssement de textes (légitimés par la forme ouverte de la répétition) pour montrer que le théâtre est un espace privilégié d'improvisation et de polyphonie intertextuelle: il intercale dans la pièce des impromptus personnels ironiques et sarcastiques renvoyant à la réalité artistique qui l'entoure (un miroir critique de Molière), en même temps qu'il fait réciter Racine, en français (le célèbre monologue de Phèdre), à Mlle Du Parc/Amélia Matta, pour rendre hommage à cette actrice portugaise, le public pouvant voir, projetées sur le fond de la scène, des photos allusives à ses rôles les plus marquants (une mise en abyme vertigineuse).

8 L'artifice est, ainsi, constamment exhibé sur scène et devant les spectateurs, comme dans les salons de Versailles, ne permettant jamais d'éluder le jeu théâtral : on le constate par l'intervention ponctuelle d'une voix off, sorte de souffleur (une autre voix recréée), qui fait allusion à des intertextes de Molière (Plaute, Térence), qui cite des extraits d'ouvrages critiques concernant la vie du dramaturge, sa pratique du théâtre dans le théâtre, tandis que l'acteur Molière et Mademoiselle Molière s'adonnent à des jeux érotiques dérisoires, comme s'il y avait une permanente et "naturelle» intersection d'intertextes, de fragments de fictions et de réalités, sur scène.

9 C'est justement autour de la mise en place des enjeux spéculaires du texte, c'est-à-dire des différentes voix qui se multiplient dans cette mise en scène extravagante, que Miguel Loureiro nous fait part du vertige de l'ironie avec lequel il réécrit, jusqu'à l'exhaustion, le méta-commentaire moliéresque. Au fond, c'est le principe d'encadrement de L'Impromptu qui permet au metteur en scène portugais de jouer avec la distance esthétique imposée par la pièce vis-à-vis du spectateur, soulignant la labilité de toute frontière érigée entre fiction (la pièce dans le théâtre) et réalité (la pièce sur le théâtre, écrite pour apprendre à jouer « un rôle où l'on doit parler naturellement » (Molière, 1965 : 158).

10 Comprendre la modernité de ces enjeux, ancrée dans l'instabilité des frontières entre fiction et réalité, et dans l'autoréflexion esthétique développée dans le texte du XVII ${ }^{\mathrm{e}}$ siècle, est, aussi, le principe esthétique qui marque l'écriture scénique de Ricardo Pais lorsqu'il transpose le Dom Juan de Molière sur la scène portugaise, en 2006. Comme Miguel Loureiro, le metteur en scène crée une stratégie herméneutique qui accentue la distance théâtrale, le pacte métathéâtral, le jeu de miroirs et de dédoublements inscrits in fieri dans l'original, envisageant le texte traduit par Nuno Júdice comme un "exercice de style " (Cabral et al., 2006: 17) contemporain. Il s'agit de regarder le «banquet mythique » du libertin, réponse théâtrale de Molière à la cabale des faux dévots qui interdit la représentation de Tartuffe, comme un prétexte politique et esthétique qui, comme l'Impromptu, mise sur l'autoréflexivité dramatique et sur l'idée, chère à Ricardo Pais, de précarité et de rupture du drame et de l'homme-acteur. En fait, l'autoportrait de 
Dom Juan ${ }^{10}$ et son rapport spéculaire à Sganarelle, les différents masques exhibés par le personnage (séducteur et inconstant, libertin, courtisan, hypocrite), mettent en évidence la dispersion de l'action faisant partie de l'ontologie du spectacle théâtral soutenue aussi bien par Molière ${ }^{11}$, au Grand Siècle, que par Ricardo Pais, en 2006. À cet égard, Pais, démontre d'ailleurs que la marginalité esthétique et éthique de la pièce moliéresque une pièce, affirme-t-il, « extrêmement imparfaite mais clairement construite » (Cabral et al., 2006: 15) -, conçue à partir d'un écart volontaire par rapport aux règles et à la vraisemblance classiques ${ }^{12}$, suppose une interrogation moderne sur la nature de la théâtralité, sur l'essence du personnage de théâtre. C'est pourquoi la voix de Dom Juan (et de Molière) se confond, dans les plans esthétique et éthique, avec celle du metteur en scène, ne serait-ce que par le souci qu'il exprime, tout au long de la représentation, de montrer la vacuité d'une pulsion érotique qui mène le protagoniste à se lancer dans de nouveaux cercles de vie, apparemment sans issue, traduisant le désespoir du corps face à la mort ${ }^{13}$. L'unité d'action que Molière fait en permanence basculer par l'imposition d'une structure en fuite, éparpillée (annonçant, partiellement, celle de L'Impromptu), demeure, dans la mise en scène de Ricardo Pais, une sorte de fil conducteur précaire qui s'appuie sur le parcours du protagoniste vers la mort - une fuite libertine et tragique vers la transcendance -, conjuguant, sans relâche, la voix et le corps du personnage mythique, son corps et son masque infernal (Pais, $2006: 3$ ).

D'ailleurs, Pais joue souvent, dans cette mise en théâtre baroque de la mort du libertin, avec la superposition paradoxale du corps (humain) et du non-corps (surnaturel) des acteurs qui annoncent la dé-composition finale du corps de Dom Juan (et le dénouement théâtral) : un seul et même acteur joue le rôle du Pauvre, de Dom Louis, de la Statue du Commandeur (un fantôme), un même corps et une même voix sous des habits différents, configurant des obstacles tragiques à l'impiété du protagoniste. La pluralité des voix et des corps émanant du texte du XVII ${ }^{e}$ est, de ce fait, récupérée para Ricardo Pais (ainsi que par Miguel Loureiro) pour souligner une certaine irréalité du texte théâtral («Il n’y a aucun trait de réalisme dans ce spectacle »(Cabral et al., 2006:19), affirme Pais), pour souligner ses enjeux ironiques et utopiques: la chorégraphie symbolique de deux mises en scènes en est une sorte de pari, semblant vouloir récupérer, dans les deux cas, différemment, le climat de fantaisie produit par les machines théâtrales italiennes du XVII e, dont Molière, ainsi que Louis XIV, étaient des admirateurs. La voix du texte, de la parole qui se fait corps, devient, donc, aussi, la voix de la scène.

\section{La voix de la scène (ou Molière sur scène)}

12 On revient, de nouveau, à Nuno Júdice, traducteur portugais de Dom Juan de Molière pour la mise en scène de Ricardo Pais et à la définition du théâtre qu'il suggère dans la préface "La parole et le mythe »: "Le théâtre est le désir qu'a la parole de se faire corps " (Júdice, 2006 : 7). En fait, au niveau de la " performance », Miguel Loureiro et Ricardo Pais semblent oublier le texte pour le rendre visible à travers le corps de chaque acteur et de son rapport à la scène, comme s'ils incarnaient, au xxI ${ }^{e}$ siècle, le rôle de Molière metteur en scène et acteur de la troupe du Roi, à Versailles et au Palais-Royal. L'importance que Molière accorde au détail de la mise en scène, le souci de régler le jeu de chaque acteur, de faire voir, sur scène, l'enchâssement décomposé du texte, se matérialise dans des décors qui ressemblent à des "installations $~^{14}$ artistiques néobaroques. De ce fait, les métamorphoses visibles du décor, dans le Dom Juan de Ricardo Pais, accompagnant 
l'évolution de l'intrigue vers la mort du protagoniste (la mutation transcendantale du corps), ou le mouvement circulaire de la scène de la pièce de Loureiro, faisant tourner sans fin les acteurs comme s'ils appartenaient à un carrousel de fantaisie, visent et à compléter le travail méta-théâtral exhibé dans les deux pièces du Grand Siècle et à souligner la relation instable entre réalité et fiction, voire le rapport au théâtral ${ }^{15}$, qu'elles ne cessent de suggérer.

Dans la mise en scène portugaise de Dom Juan, une plateforme en bois devient de plus en plus oblique, se ferme sur elle-même et sur les cavités sombres des trappes, au fur et à mesure que le duo maître/valet entame une nouvelle étape, que Dom Juan se lance dans la recherche du désir matériel et érotique du corps, jusqu'à la mort inévitable et spectaculaire : la plateforme devient à demi oblique, au début de l'Acte III, pour souligner le motif libertin du double déguisement tragi-comique - Dom Juan, « en habit de campagne ", Sganarelle, "en médecin » (Molière, 1965: 380); le mouvement vers l'oblique s'accentue lorsque la statue du commandeur ou Elvire sortent d'une porte ou gouffre noir pour annoncer la mort de Dom Juan, voire le passage à une dimension transcendantale du corps. En outre, les effets de miroir se multiplient, suggérant l'ondulation polyphonique du texte, l'instabilité de la vie, du réel, et de sa mise en théâtre: les trappes s'ouvrent et se referment sans cesse créant des spécularités plastiques qui mettent en évidence le caractère théâtral du duo maître/valet, leur complicité ambigüe servant d'enjeux implicite à la critique de l'hypocrisie sociale et religieuse, motif majeur du texte au XVII ${ }^{e}$, prétexte métaphysique de la mise en scène au $\mathrm{XXI}^{\mathrm{e}}$ siècle.

De même, dans la mise en scène portugaise de L'Impromptu, le miroir se fait symbole du méta-théâtre et décor presque unique: une série de miroirs à grandes dimensions, disposés aléatoirement sur scène, sont déplacés par les acteurs eux-mêmes, qui tournent en rond avec eux, réfléchissant leurs habits de comédiens du $\mathrm{XVII}^{\mathrm{e}}$ aux couleurs excentriques. Visant à reproduire la voix plurielle et ironique du texte, la multiplication extravagante d'images révèle la labilité de la répétition projetée en kaléidoscope des corps et des couleurs, le théâtre pouvant être compris comme objet d'essai et d'expérimentation, forme extrême de spécularité, soulignant l'art du dédoublement scénique de l'impromptu, du théâtre dans le théâtre et de la commedia dell'arte réinvité par Molière ${ }^{16}$ et par Miguel Loureiro. Le mélange coloré de l'acteur et de son double propose, ainsi, en 2016, une lecture moderne de l'esthétique de la comédie-miroir qui séduisait le dramaturge du Roi et Louis xIV lui-même, tout en essayant de reproduire, sur scène, l'idée baroque du spectacle total.

Il semble, donc, logique, partant de cette notion théorique de 'spectacle total', (Fleck, 2007) que les deux mises en scène contemporaines de Molière parviennent à définir, in fieri, une synthèse poétique (le spectacle total) qui fait signifier les voix et les corps dramatiques, sur scène, au-delà de la scène, par un exercice circonstancié du rythme (qui est aussi écriture, parole improvisée). Avant que l'impromptu ne commence, Miguel Loureiro fait défiler, à travers le parterre jusqu'au-devant de la scène, Molière-acteur, Mlle du Parc, Mlle Béjart, Mme Molière et un violoniste qui restera toujours sur scène, sur la droite (presque hors scène), dont la musique associe le rythme aux couleurs et au langage des personnages, formant un certain code des mots (l'accord musical sert, sans désemparer, d'euphémisme pour substituer le mot « roi »). Sa musique accompagnera, aussi, les danses de cour successives des acteurs, suggérées par Du Croisy, l'acteur-poète, et servira à reproduire, en dérision ironique, les comédies-ballets composées par le 
dramaturge, à Versailles, pour le plaisir du regard, cette extravagance chorégraphique forçant le dispositif du dédoublement des voix.

De même, l'entrée sur scène de Dom Juan, habillé en costume doré, est accompagnée d'un clarinettiste habillé en noir qui le suit, comme une ombre ou un double, jouant en crescendo musical, jusqu'au dénouement, accentuant l'instabilité ontologique et idéologique du libertin (un personnage en rupture). Par ailleurs, un rythme de jazz non cadencé, en rupture, s'allie au rythme du bruit cadencé de l'ouverture et de fermeture des trappes, aux brefs moments d'un air d'opéra tragique chanté par Elvire, lorsqu'elle annonce la mort théâtrale de Dom Juan. L'ensemble rythmique accentue, ainsi, les enjeux polémiques de la distance théâtrale et, ce faisant, la polyphonie actuelle du théâtre de Molière. La poétique du spectacle total, de la fête baroque se fait poétique du rythme des corps et des voix dans le paysage protéiforme, décentré ${ }^{17}$, de la scène contemporaine qui accueille Molière.

Deux images-cadres, correspondant aux dernières minutes de la représentation de L'Impromptu de Versailles, font la synthèse de ces manipulations des voix. Dans le premier cadre, située à la scène 7 (Molière, 2016: 40), tous les personnages-acteurs dansent à un rythme vertigineux, la scène tourne, les lumières à couleurs variées s'impriment sur les vêtements colorés des acteurs, Béjart, le nécessaire, court entre le devant de la scène et le parterre annonçant l'arrivée du Roi, les acteurs hurlent et lancent des cris hystériques car ils sont incapables de représenter la pièce qu'ils n'ont pas réussi à répéter. La parole des acteurs est, ainsi, substituée par le mouvement de leurs corps et par la musique, comme si le théâtre ne pouvait être qu'une danse dérisoire et carnavalesque. Le deuxième cadre correspond à la fin de la mise en scène, annulant le texte, en traduction, du dramaturge (Molière, 2016: 42), et isolant Molière-acteur sur scène, seul parmi les grands miroirs, disant, en français, « la comédie ne peut pas bien finir », au son de la musique jouée par le violoniste. En même temps, un mécanisme scénique le fait descendre lentement au milieu de la fumée. Les deux moments-cadre nous montrent, en fait, que Miguel Loureiro (ainsi que Ricardo Pais et Molière lui-même) se sont servis de l'écriture dramatique de JeanBaptiste Poquelin pour ne dévoiler qu'en partie le mystère de l'incarnation scénique : la mort invraisemblable de Dom Juan, ainsi que l'impromptu à jamais accompli, soulignent, dans le cadre du déplacement historique du discours et du déplacement de la scène baroque à la scène néobaroque, la survie de l'utopie de l'écriture (et de la voix) dans l'espace ouvert du théâtre, du théâtre dans le théâtre et du théâtre sur le théâtre. Aujourd'hui, comme au XVII ${ }^{e}$ siècle, la parole s'approprie le corps et se fait spectacle des voix sur scène.

\section{BIBLIOGRAPHIE}

ANDREWS, Richard (2005). « Molière, commedia dell'arte, and the question of influence in early modern Europe theatre ", Modern Language Review, n100, 2, pp. 444-463.

BABY, Hélène (2001). La tragi-comédie de Corneille à Quinault. Paris : Klincksieck. 
BABY et VIALA (2016), « Le XVII ${ }^{\mathrm{e}}$ siècle ou l'institution du théâtre » in Alain Viala (org.). Le théâtre en Frances. Paris : PUF, pp. 161-249.

BIONDA, Romain (2017). « La vérité du drame : lire le texte dramatique (Dom Juan) », Poétique, nº 181 (1), pp. 67-82.

BOURQUI, Claude et VINTI, Claudio (2003). Molière à l'école italienne : le 'lazzo' dans la création moliéresque. Torino : L'Harmatta Italia ; Paris : L'Harmattan.

CABRAL, Patrícia et al. (2006). « Discurso sobre um tarado 'aditivo'. ». D. João. Manual de Leitura : 17. CHAOUCHE, Sabine (dir.) (2010). Le 'théâtral' de la France d'Ancien Régime. De la représentation de soi à la représentation scénique. Paris : Champion.

CIVARDI, Jean-Marc (2011). « Les querelles dramatiques sont-elles un genre ? », CdG - Les Cahiers du Gadges, n9, pp. 167-209.

DANDREY, Patrick (2014). La guerre comique: Molière et la Querelle de l'Ecole des Femmes. Paris : Hermann Éditeurs.

DотоLI, Giovanni (2001). Littérature et société en France au XVII siècle : volume III. Fasano ; Paris : Schena editore : Presses de l'Université de Paris-Sorbonne.

DUPRAT, Anne (2009). Vraisemblances. Poétiques et théorie de la fiction, du Cinquecento à Jean Chapelain (1500-1670). Paris : Champion.

FORESTIER, Georges (1988). Esthétique de l'identité dans le théâtre français : 1550-1680. Les déguisements et ses avatars. Genève : Droz.

FLECK, Stephen H. (2007). « Représentation et spectacle dans les comédies-ballets : de la représentation du monde à la création du spectacle total » in Rainer Zaiser (org.). L'âge de la représentation : l'art du spectacle au XVII siècle. Tübingen : G. Narr, pp. 195-206.

FORESTIER, Georges (1990). Molière en toutes lettres. Paris : Bordas.

FORESTIER, Georges et NÉRAUDAU, Jean-Pierre (dir.) (1995). Un Classicisme ou des Classicismes ?. Pau : Publications de l'Université de Pau.

FORESTIER, Georges (1996). Le théâtre dans le théâtre sur la scène française du XVIIe siècle. Genève :

Droz.

HENIN, Emmanuelle (org.) (2010). Les querelles dramatiques à l'âge classique (XVII ${ }^{e}$-XVIII ${ }^{e}$ siècles).

Louvain-Paris-Walpole : Éditions Peeters.

HORVILLE, René (1972). ‘Dom Juan' de Molière. Une dramaturgie de rupture. Paris : Larousse.

JÚDICE, Nuno (2006). «A Palavra e o Mito », in Molière, D. João ou O Banquete de Pedra. Porto : Campo das Letras, pp. 11-15.

MAZOUER, Charles (2006). Molière et ses comédies-ballets. Paris : Champion.

MEschonnic, Henri (1973). Pour la Poétique II. Épistémologie de l'écriture. Poétique de la Traduction.

Paris : Gallimard.

MESCHONNIC, Henri (1986). « Alors la traduction chantera », Revue d'Esthétique (La Traduction), no12, pp. 75-80.

MOLIÈRE (1965). CEuvres Complètes 2. Paris : Garnier-Flammarion.

MOLIÈre (2006). D. João ou O Banquete de Pedra. Porto : Campo das Letras. 
MOLIÈRE (2016). O Impromptu de Versalhes. Lisboa : TNDM II \& Bicho-do-Mato.

PAIS, Ricardo (2006). « O festim de pedra ». D. João. Manual de Leitura : p. 3.

RIBEIRO, João Mendes (2006). «Paisagem mental ». D. João. Manual de Leitura : p. 5.

SCHÉRER (2014). La dramaturgie classique (nouvelle édition). Paris: Armand Colin.

SPIELMANN, Guy (2001). «Pour une syntaxe du spectaculaire au XVII ${ }^{\mathrm{e}}$ et au XVIII ${ }^{\mathrm{e}}$ siècles » in MarieFrance Wagner et Claire Le Brun-Gouanvie (eds). Les arts du spectacle au théâtre (1550-1700). Paris : Champion, pp. 219-260.

TEIXEIRA ANACLETO, Marta (2015). « Décors discontinus : avatars du jeu théâtral moliéresque sur la scène portugaise au XxI ${ }^{\mathrm{e}}$ siècle » in Pierre Zoberman (ed.). Interpretation in/of Seventeenth Century. Cambridge: Cambridge Scholars Publishing, pp. 397-410.

\section{NOTES}

1. - Nous traduisons.

2. - Voir, au sujet des "Querelles» qui ont marqué le parcours dramatique de Molière et la conception dramatique même de ses pièces, entre autres : Baby et Viala, 2016 : 161-249; Civardi, 2011 : 167-209 ; Forestier, 1990 ; Forestier et Néraudau, 1995 ; Henin, 2010 ; Scherer, 2014.

3. - Expression utilisée au sens que lui donne H. Meschonnic lorsqu'il considère que l'historicité des discours fait la critique (Meshonnic, 1986 : 90).

4. - Pour mieux comprendre le contexte particulier de cette querelle et l'histoire de la séquence des textes dramatiques, voir Dandrey, 2014.

5. - « MOLIÈRE.- De grâce mettons-nous ici, et puisque nous voilà tous habillés, et que le Roi ne doit venir de deux heures, employons ce temps à répéter notre affaire, et voir la manière dont il faut jouer les choses. (...)Et n'ai-je à craindre que le manquement de mémoire ? Ne comptez-vous pour rien l'inquiétude d'un succès qui ne regarde que moi seul ? Et pensez-vous que ce soit une petite affaire, que d'exposer quelque chose de comique devant une assemblée comme celle-ci? que d'entreprendre de faire rire des personnes qui nous impriment le respect, et ne rient que quand ils veulent? Est-il auteur qui ne doive trembler, lorsqu'il en vient à cette épreuve ? Et n'est-ce pas à moi de dire que je voudrais en être quitte pour toutes les choses du monde?» (Molière, $1965: 148$ ).

6. - « MOLIÈRE. - J'avais songé une comédie, où il y aurait eu un poète que j'aurais représenté moi- même, qui serait venu pour offrir une pièce à une troupe de comédiens nouvellement arrivés de la campagne. (...) Et le poète : «Comment, vous appelez cela réciter ? C'est se railler ; il faut dire les choses avec emphase. Écoutez-moi. (Imitant Montfleury, excellent acteur de l'Hôtel de Bourgogne.) » (Molière, 1965 : 151).

7. - « MADEMOISELLE DE BRIE. - «Vous voulez bien, Mesdames, que nous vous donnions en passant la plus agréable nouvelle du monde. Voilà Monsieur Lysidas qui vient de nous avertir qu'on a fait une pièce contre Molière, que les grands comédiens vont jouer. / MOLIÈRE. - « Il est vrai, on me l'a voulu lire, et c'est un nommé Br... Brou... Brossaut qui l'a faite. / DU CROISY. «Monsieur, elle est affichée sous le nom de Boursaut, mais à vous dire le secret, bien des gens ont mis la main à cet ouvrage, et l'on en doit concevoir une assez haute attente. Comme tous les auteurs, et tous les comédiens regardent Molière comme leur plus grand ennemi, nous nous sommes tous unis pour le desservir ; chacun de nous a donné un coup de pinceau à son portrait, mais nous nous sommes bien gardés d'y mettre nos noms; il lui aurait été trop glorieux de succomber aux yeux du monde, sous les efforts de tout le Parnasse ; et pour rendre sa défaite plus 
ignominieuse, nous avons voulu choisir tout exprès un auteur sans réputation. » (Molière, 1965 : 161).

8. - Les ouvrages de Georges Forestier démontrent brillamment l'importance singulière de la méta-théâtralité dans la poétique dramatique du XVII ${ }^{\mathrm{e}}$ siècle et son incidence dans le théâtre de Molière (Forestier 1988 ; 1996).

9. - Lors de la première de la pièce, on a distribué aux spectateurs un Guide de Lecture (Manual de Leitura), publié pour l'occasion. On s'en servira à plusieurs reprises dans cet article, en traduisant les textes en français.

10. - «DOM JUAN.- Quoi ? tu veux qu'on se lie à demeurer au premier objet qui nous prend, qu'on renonce au monde pour lui, et qu'on n'ait plus d'yeux pour personne? La belle chose de vouloir se piquer d'un faux honneur d'être fidèle, de s'ensevelir pour toujours dans une passion, et d'être mort dès sa jeunesse, à toutes les autres beautés qui nous peuvent frapper les yeux : non, non, la constance n'est bonne que pour des ridicules, toutes les belles ont droit de nous charmer, et l'avantage d'être rencontrée la première, ne doit point dérober aux autres les justes prétentions qu'elles ont toutes sur nos cœurs. Pour moi, la beauté me ravit partout, où je la trouve; et je cède facilement à cette douce violence, dont elle nous entraîne. » (Molière, 1965 : 360).

11. - Voir le concept de «dramaturgie de rupture » développé par R. Horville à propos de Dom Juan de Molière (Horville, 1972).

12. - Voir, au sujet de la problématique de la vraisemblance dans la poétique dramatique du XVII et de ses enjeux dans le contexte de la production de la tragi-comédie, tel que Molière conçoit Dom Juan, les études suivantes (entre autres) : Baby, 2001 ; Bionda, 2017 ; Duprat, 2009.

13. - On reprend, dans cet article, l'argumentation développée dans un texte de 2015 où il a été question d'analyser parallèlement, à partir de la notion de «décor discontinu ", cette représentation du D. Juan de Ricardo Pais et la mise en scène de l'Avare, par Rogério de Carvalho, en 2009 (Teixeira Anacleto, 2015 : 397-410).

14. - C'est ainsi que João Mendes Ribeiro, le décorateur choisi par R. Pais, nomme le décor conçu par lui-même, insistant, en même temps, sur la notion de " paysage mental » (Ribeiro, $2006: 5$ ).

15. - Plusieurs études furent consacrées, dans les dernières années, à l'étude de ce concept du «théatral » au XVII ${ }^{\mathrm{e}}$ siècle, quelques-unes faisant justement allusion à son rapport aux pièces de Molière. Voir, entre autres : Chaouche, 2010 ; Mazouer, 2006 ; Spielmann, 2001.

16. - Sur les rapports entre la dramaturgie de Molière, voir, entre autres, les études récentes de Richard Andrews (Andrews, 2005), Claude Bourqui et Claudio Vinti (Bourqui et Vinti, 2003), Giovanni Dotoli (Dotoli, 2001).

17. - Au sens où H. Meschonnic utilise, depuis 1973, le concept de «décentrement » et de « rythme » à propos de la théorie et épistémologie de la traduction et du langage (Meschonnic, 1973).

\section{RÉSUMÉS}

Représenter Molière, sur la scène contemporaine portugaise, relève d'un défi méta-théâtral, la scène devenant un lieu de création utopique des voix, des corps, des objets qui faisaient sens à Versailles. Si le D. João de Ricardo Pais (2006) s'exhibe en spectacle comique et tragique du libertinage érudit $\mathrm{du} \mathrm{XVII}^{\mathrm{e}}$ siècle, la mise en scène de L'Impromptu de Versailles, par Miguel 
Loureiro (2016), exploite les rapports ontologiques entre l'illusion théâtrale et les enjeux néobaroques de la représentation. En partant des notions de «déplacement historique des discours " et d'« invention de l'oralité qui transforme l'oralité », présentées par Meschonnic à propos de la poétique de la (ré)écriture, on analysera le sens du « décentrement » des voix dans ces deux représentations contemporaines de Molière, en démontrant comment la modernité du dramaturge est assurée par des relectures actuelles multiples de la structure méta-dramatique de ces pièces.

To stage a Molière play in Portugal nowadays poses a metatheatrical challenge as the stage itself becomes a space for the utopian recreation of voices, bodies and objects which once made sense in Versailles. D. João, by Ricardo Pais (2006) becomes a tragi-comic spectacle of seventeenthcentury erudite libertinism, while Miguel Loureiro's mise-en-scène of L'Impromptu de Versailles (2016) delves into the ontological relations between theatrical illusion and the neobaroque challenges of the performance. Starting from Meschonnic's notions of "historical displacement of discourses" and "the invention of orality that transforms orality" which he developed in connexion with the poetics of (re)writing, this paper addresses the meaning of the «decentering" of the voices in the two contemporary renderings of Molière's plays, showing that the playwright's modernity is assured by multiple modern-day rereadings of the metadramatic structure of these plays.

\section{INDEX}

Mots-clés : Molière, Pais (Ricardo), Loureiro (Miguel), méta-théâtre, réécriture

Keywords : Molière, Pais (Ricardo), Loureiro (Miguel), Meta-theatre, rewriting

\section{AUTEUR}

MARTA TEIXEIRA ANACLETO

Faculté des Lettres/CLP-Université de Coimbra marta[at]fl.uc.pt 